\title{
Imaging Plasmons with Sub-2 nm Spatial Resolution via Tip-Enhanced Four-Wave Mixing
}

\author{
Chih-Feng Wang and Patrick Z. El-Khoury*
}

Physical Sciences Division, Pacific Northwest National Laboratory, P.O. Box 999, Richland, WA 99352

patrick.elkhoury@pnnl.gov 

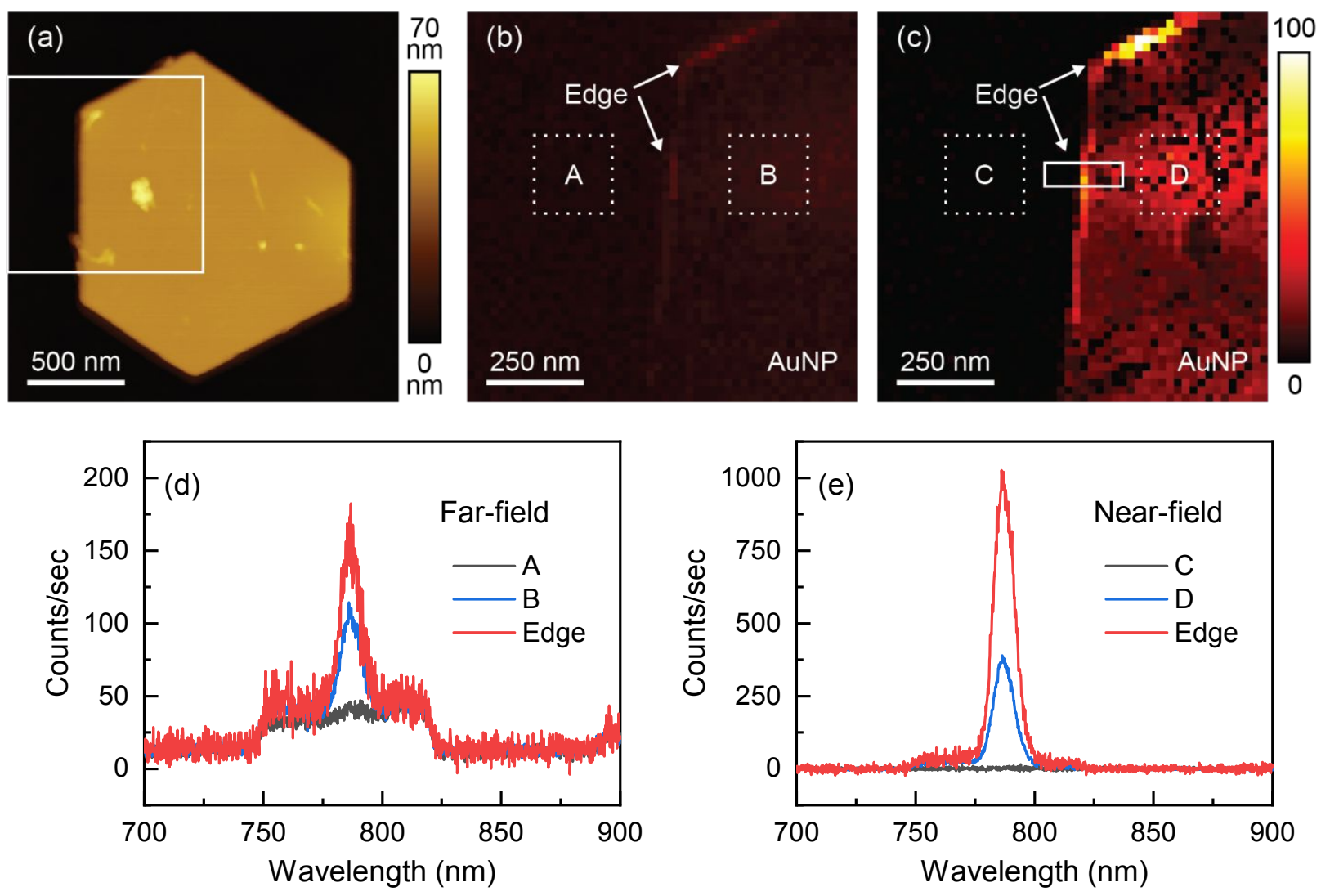

Figure S1. 4WM nano-imaging and nano-spectroscopy of a plasmonic gold nanoplate. Shown are correlated topographic (a) and 4WM images (b) and (c). Note that far- (b) and near-field (c) images were simultaneously recorded (see main text for more information). Signals contained within the areas highlighted in panels (b) and (c) are averaged and plotted in (d) for far-field and (e) for near-field spectra. Note that the scale bar in the upper-right corned of the figures is in counts/0.1 s. 


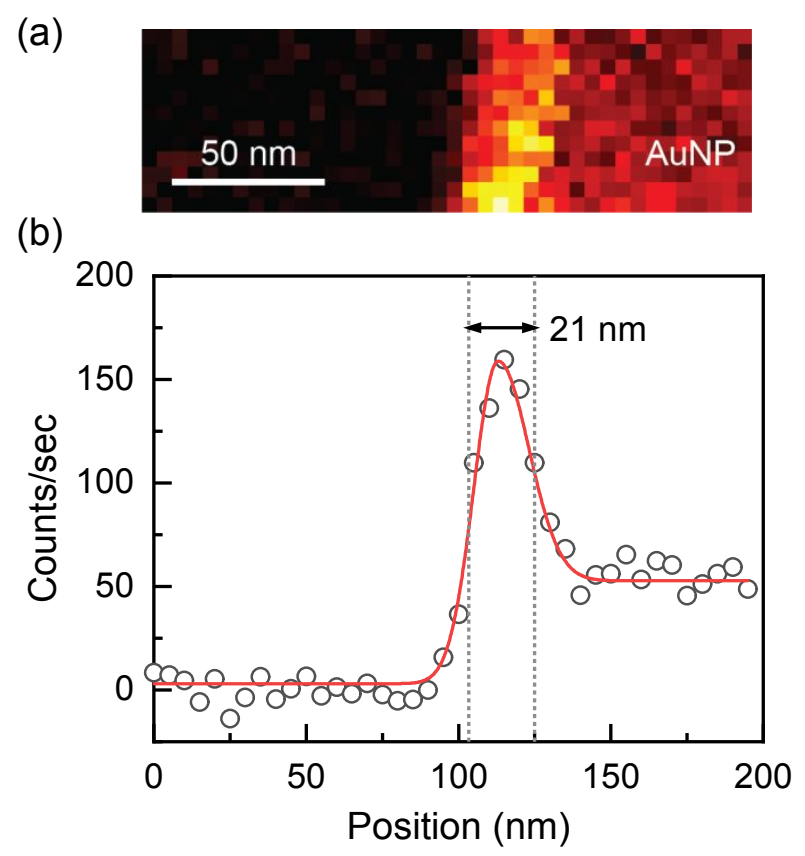

Figure S2. High-resolution 4WM nanoscopy of the edge of the nanoplate shown in Figure S1. Both the image and an averaged (over the y-direction) cross-sectional cut are shown. The reader is referred to the main text and reference 16 (in the main text) for more information. 

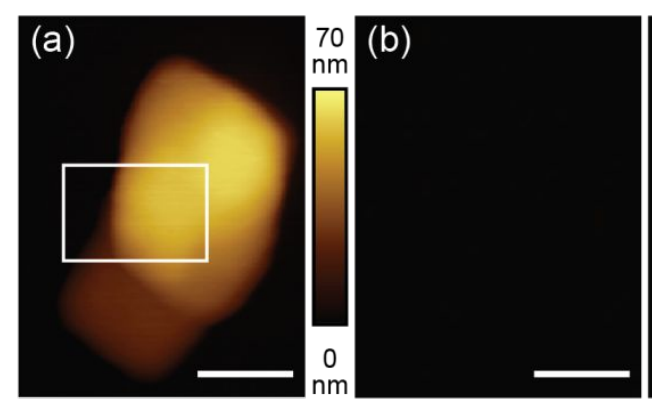

(e)

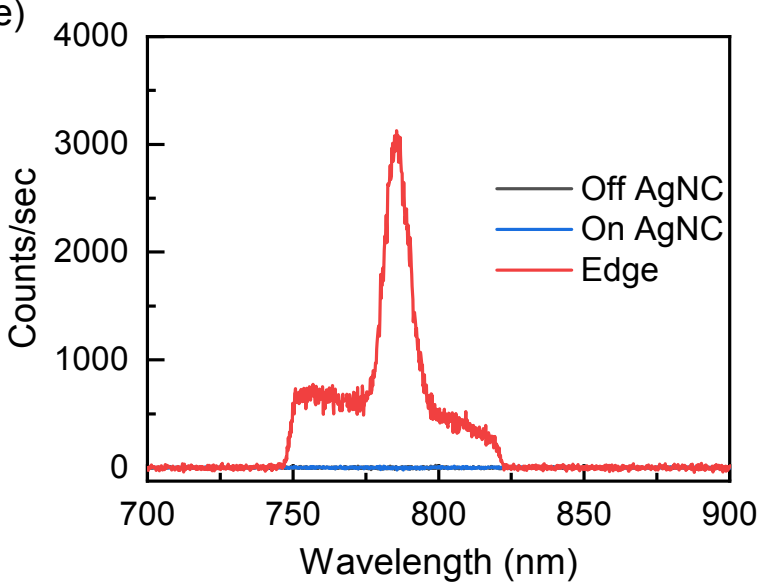

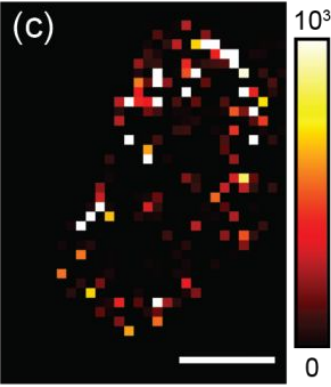

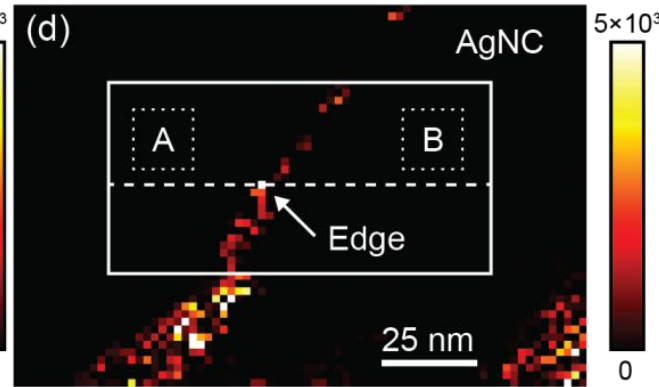

(f)

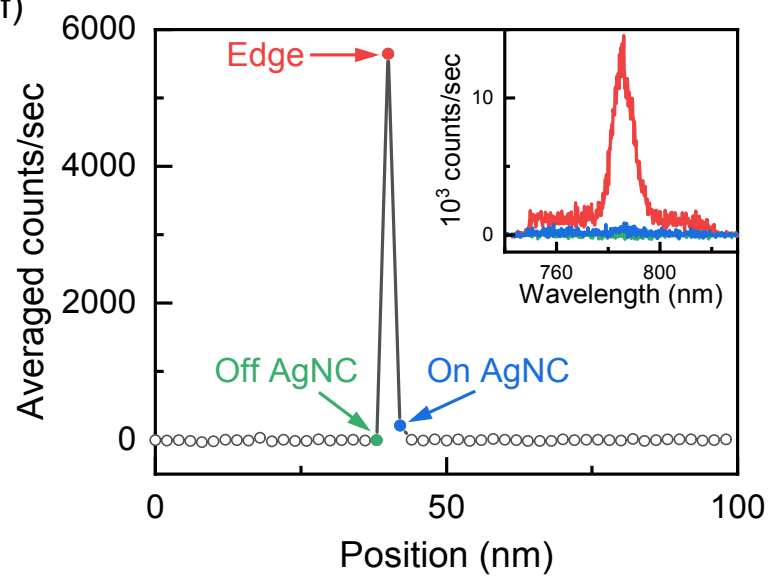

Figure S3. 4WM nano-imaging and nano-spectroscopy of a plasmonic silver nanocube. Shown are correlated topographic (a) and 4WM images (b), (c), and (d). Note that far- (b) and near-field (c and d) images were simultaneously recorded (see main text for more information). Also note that (d) is in essence a zoomed-in/higher resolution map of the edge of the cube shown in (c). Signals contained within the areas highlighted in panel (d) are averaged and plotted in (e). Note that region A in (d) is offparticle (denoted as 'Off AgNC in (e)), whereas region B in (d) is on-particle (denoted as 'On AgNC in (e)). In (f), a cross-sectional cut taken along the dashed line shown in (d) is plotted, and single pixel offparticle, on-particle, and on-edge spectra are shown. In Figure 4 of the main text, several additional cross-sectional line cuts (akin to their analogues in (f)) are shown. 

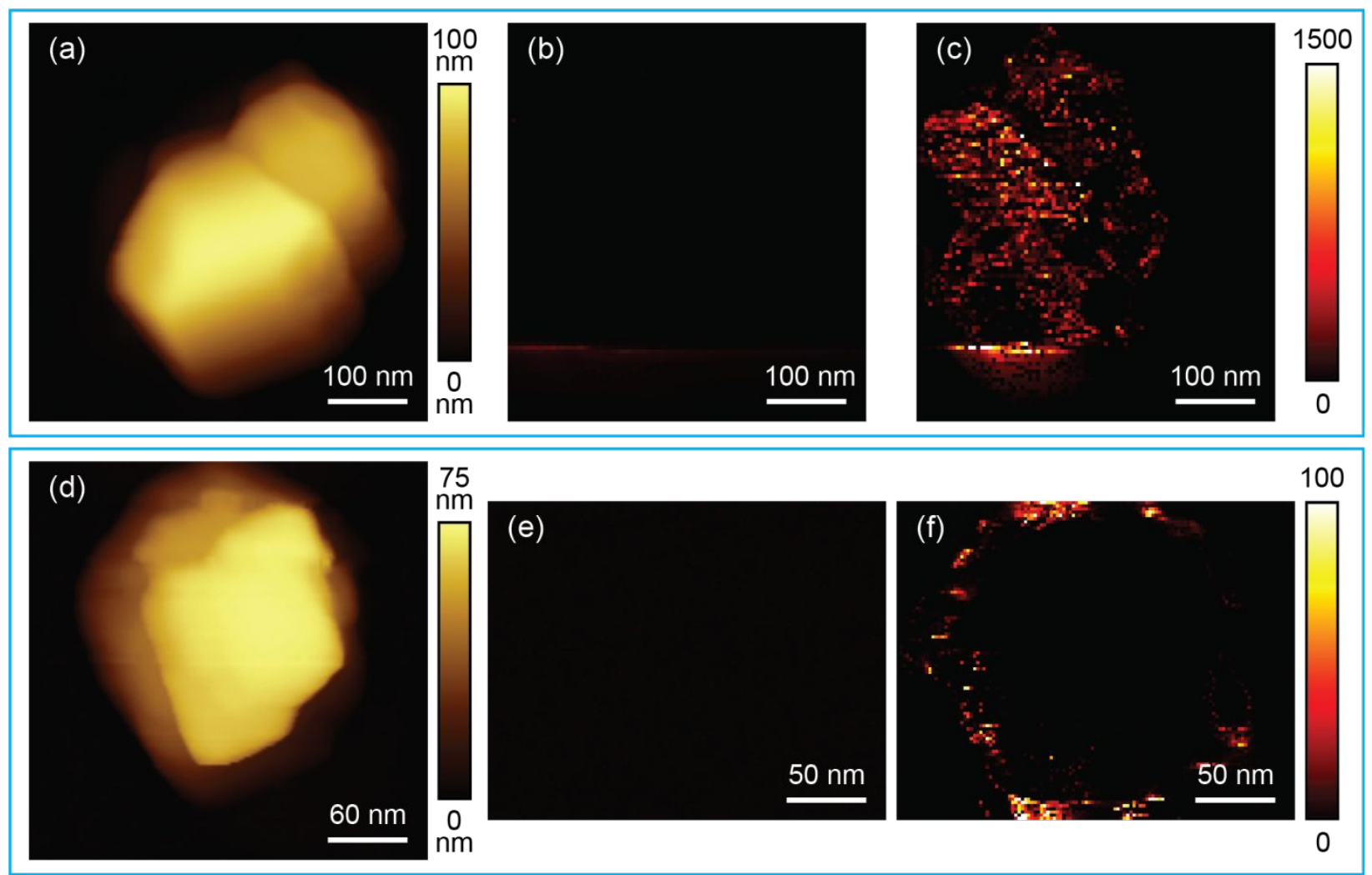

Figure S4. 4WM nano-imaging and nano-spectroscopy of two different plasmonic silver nanocubes. These images were recorded using two different (blunt/distorted) tips. Shown are correlated topographic (a,d) and 4WM images (b), (c), (e) and (f). Note that far- (b,e) and near-field (c,f) images were simultaneously recorded (see main text for more information). The near-field images are evidently contaminated by the tip shape, as evidenced from the simultaneously recorded AFM maps. 